\title{
Long term survival with stereotactic ablative radiotherapy (SABR) versus thoracoscopic sublobar lung resection in elderly people: national population based study with propensity matched comparative analysis
}

\author{
Subroto Paul, ${ }^{1}$ Paul C Lee, ${ }^{2}$ Jialin Mao, ${ }^{3}$ Abby J Isaacs, ${ }^{3}$ Art Sedrakyan ${ }^{3}$
}

1Department of Cardiothoracic

Surgery, RWJ Barnabas Health

System, West Orange, NJ, USA

2Department of Thoracic

Surgery, New York Presbyterian

Hospital/Queens, NY, USA

${ }^{3}$ Department of Healthcare

Policy and Research, Weill

Cornell Medical College and

New York Presbyterian Hospital/

New York, 402 E 67th Street,

New York, NY, 10065, USA

Correspondence to:

A Sedrakyan

ars2013@med.cornell.edu

Additional material is published

online only. To view please visit

the journal online.

Cite this as: BMJ 2016;354:i3570 http://dx.doi.org/10.1136/bmj.i3570

Accepted: 24 June 2016

\section{ABSTRACT}

OBJECTIVES

To compare cancer specific survival after thoracoscopic sublobar lung resection and stereotactic ablative radiotherapy ( $S A B R$ ) for tumors $\leq 2 \mathrm{~cm}$ in size and thoracoscopic resection (sublobar resection or lobectomy) and SABR for tumors $\leq 5 \mathrm{~cm}$ in size.

DESIGN

National population based retrospective cohort study with propensity matched comparative analysis.

SETTING

Surveillance, Epidemiology, and End Results (SEER) registry linked with Medicare database in the United States.

\section{PARTICIPANTS}

Patients aged $\geq 66$ with lung cancer undergoing $S A B R$ or thoracoscopic lobectomy or sublobar resection from 1 Oct 2007 to 31 June 2012 and followed up to 31 December 2013.

\section{MAIN OUTCOME MEASURES}

Cancer specific survival after SABR or thoracoscopic surgery for lung cancer.

RESULTS

690 (275 (39.9\%) SABR and 415 (60.1\%) thoracoscopic sublobar lung resection) and 2967 (714 (24.1\%) SABR and 2253 (75.9\%) thoracoscopic resection) patients were included in primary and secondary analyses. The average age of the entire cohort was 76. Follow-up of the entire cohort ranged from 0 to 6.25 years, with an average of three years. In the primary analysis of patients with tumors sized $\leq 2 \mathrm{~cm}, 37$ (13.5\%) undergoing SABR and 44 (10.6\%) undergoing thoracoscopic sublobar resection died from lung cancer, respectively. The cancer specific survival diverged after one year, but in the matched analysis (201 matched patients in each group) there was no significant difference between the groups (SABR $v$ sublobar lung resection mortality: hazard ratio 1.32 ,

\section{WHAT IS ALREADY KNOWN ON THIS TOPIC}

Stereotactic body radiotherapy (SABR) can provide acceptable local control in patients with medically operable and inoperable early stage non-small cell lung cancer It is unknown if survival after SABR is comparable with survival after minimally invasive surgical resection, when postoperative morbidity is reduced

\section{WHAT THIS STUDY ADDS}

Patients, especially those with larger tumors, who undergo thoracoscopic resections might have improved cancer specific survival compared with those undergoing SABR for early stage non-small lung cancer

95\% confidence interval 0.77 to $2.26 ; \mathrm{P}=0.32$ ). Estimated cancer specific survival at three years after SABR and thoracoscopic sublobar lung resection was $82.6 \%$ and $86.4 \%$, respectively. The secondary analysis (643 matched patients in each group) showed that thoracoscopic resection was associated with improved cancer specific survival over SABR in patients with tumors sized $\leq 5 \mathrm{~cm}$ (SABR $v$ resection mortality: hazard ratio $2.10,1.52$ to 2.89 ; $\mathrm{P}<0.001$ ). Estimated cancer specific survival at three years was $80.0 \%$ and $90.3 \%$, respectively.

\section{CONCLUSIONS}

This propensity matched analysis suggests that patients undergoing thoracoscopic surgical resection, particularly for larger tumors, might have improved cancer specific survival compared with patients undergoing SABR. Despite strategies used in study design and propensity matching analysis, there are inherent limitations to this observational analysis related to confounding, similar to most studies in healthcare of non-surgical technologies compared with surgery. As the adoption of SABR for the treatment of early stage operable lung cancer would be a paradigm shift in lung cancer care, it warrants further thorough evaluation before widespread adoption in practice.

\section{Introduction}

Lung cancer is the leading cause of cancer death worldwide. ${ }^{12}$ It is hoped that the detection of early stage lung cancer will improve with the adoption of screening programs with computed tomography of the chest in patients at high risk, with concomitant improvement in patient survival as shown by the National Lung Screening Trial (NLST). ${ }^{34}$ The best choice of treatment for these often older patients with comorbidities, however, is currently in question. Surgical lobectomy, whether performed by thoracotomy or by minimally invasive thoracoscopic or robotic approaches, is generally believed to offer the best survival rates in appropriately staged patients. ${ }^{56}$ Stereotactic body radiotherapy (SBRT or SABR for stereotactic ablative radiotherapy) can provide acceptable local control in patients with medically operable and inoperable stage I non-small cell lung cancer. ${ }^{7-14}$ SABR, used in several fractions, delivers focused biologically effective doses of radiation exceeding $100 \mathrm{~Gy}$ to the tumor. The radiation dose is delivered precisely to avoid damage to nearby structures in the thorax. SABR delivered in a few outpatient treatments has the potential to offer acceptable survival without the incisions, 
morbidity, or need for inpatient treatment associated with surgery. Several phase II prospective studies, population based studies, retrospective propensity matched analyses, and pooled analyses of incomplete phase III randomized controlled trials reported that SABR was associated with overall survival similar to that after surgery for stage I non-small cell lung cancer. ${ }^{14-17}$ A major limitation of these studies was that SABR was compared with lung resection through thoracotomy whereas a more appropriate present day comparison is arguably with minimally invasive lung resection. Many of the risks of surgery arise from perioperative morbidity, which can be mitigated by minimally invasive techniques. ${ }^{518-26}$

Three phase III randomized studies were initiated to compare SABR with surgery in patients with early stage non-small cell lung cancer (the STARS trial (NCT00840749), the ROSEL trial (NCT00687986), and the ACOSOG Z4099 trial (NCT01336894)). ${ }^{14-17}$ All were closed early because of poor accrual. Given the paradigm shift in lung cancer care presented by SABR, four trials are currently being planned to compare SABR and surgery: STABLEMATES (NCT01622621), SABRETOOTH (NCT02629458), POSTILV (NCT01753414), and VALOR (CSP \#2005). ${ }^{27-30}$ None of these trials requires minimally invasive surgery, and only STABLEMATES compares SABR with sublobar resection (less than a lobectomy), which is often the only resection that older patients with comorbidities can tolerate. Surgeons and other providers might be reluctant to accept the results of these trials unless enough patients undergo minimally invasive resection.

The current place of stereotactic ablative radiotherapy in the treatment of primary non-small cell lung cancer is contentious. ${ }^{31}$ Without comparative data from modern minimally invasive techniques, no inference can be drawn about relative effectiveness of treatments. A comparison between SABR and sublobar (segmental or wedge) resection or lobectomy can be made within Surveillance, Epidemiology and End Results (SEER)-Medicare data. SEER-Medicare captures overall and cancer specific survival and is the only national cohort in the United States that captures both cancer specific information and data on long term mortality in patients aged $>65$. Analysis of these data to determine comparative effectiveness could provide some clarity to the appropriateness of SABR as well as help design and power future randomized trials.

\section{Methods}

\section{Data source}

The current observational cohort study used the Surveillance, Epidemiology and End Results (SEER) dataset that includes patient demographics, cancer diagnoses and treatment related information, and cause of death. ${ }^{3233}$ The SEER-Medicare dataset consists of registry data linked to Medicare hospital, outpatient, physician, home health, and hospice claims. Medicare insures about $97 \%$ of people aged $>65$ in the US, which allows about $93 \%$ of that population in the SEER registry to be linked to the Medicare enrolment file. ${ }^{33}$

\section{Study cohort}

From Medicare inpatient, outpatient, and carrier file databases we identified patients who underwent SABR or lung resection (open or video assisted thoracoscopic sublobar resection or lobectomy) from 1 October 2007 to 30 June 2012 in the time period from one month before to six months after diagnosis of a first primary non-small cell lung cancer (detailed codes are in table A, appendix 1). These years were chosen because many of the detailed ICD-9-CM (international classification of diseases, ninth revision, clinical modification) codes become available on 1 October 2007. The definitive surgery was defined based on the most extensive procedure recorded in Medicare claims. To ensure completeness of data, we included only patients aged $>66$ who were continuously enrolled in Medicare Part A and Part B and not enrolled in a health maintenance organization in the year before the procedure.

We further restricted the study cohort to those who had a stage I (T1 or T2, NO, M0) cancer, with tumor size $\leq 5 \mathrm{~cm}$. Patients who had multiple cancers or a second diagnosis of lung cancer in SEER were excluded. Because histology was included as an important variable in the analyses, we excluded patients without pathological confirmation. In addition, we excluded patients who received chemotherapy before SABR or lung resection. Appendix 2 shows inclusion and exclusion criteria.

Patient survival data was complete up to 31 December 2013 (defined as the end of the study). Follow-up time for the entire cohort ranged from 0 to 6.25 years. The primary analysis focused on those who underwent SABR or thoracoscopic sublobar resection (wedge or segmental resections) for a tumor sized $\leq 2 \mathrm{~cm}$. In a secondary analysis we compared patients who underwent SABR with those who underwent thoracoscopic resection (wedge or segmental resections or lobectomy) for a tumor sized $\leq 5 \mathrm{~cm}$.

\section{Outcomes}

Overall survival was defined as time until death from any cause, with patients censored at the end of the study (31 December 2013). SEER provides information about disease specific cause of death, allowing us to determine cancer specific survival (net survival from lung cancer in our study if there are no other causes of death); in this case patients were additionally censored at the time of a non-disease related death. The periodicity of radiologic imaging (chest computed tomography or positron emission tomography or positron emission tomography combined with computed tomography (PET/PET-CT)) was also determined with CPT (American Medical Association, current procedural terminology) (table A, appendix 1) within the first year and the first two years after the procedure.

\section{Variables}

We categorized patients by cancer specific, demographic, and surgery specific variables provided in the SEER registry, including stage (Derived AJCC T Stage Group, 6th ed), histology (adenocarcinoma, squamous, 
other), tumor grade, tumor size (mm), age on date of procedure, year of procedure, sex, race, marital status at diagnosis, metropolitan area (metropolitan, non-metropolitan), registry region (Midwest, Northeast, South, West), and census tract per capita income (quarters of income distribution). Additionally, we identified patients' comorbidities within a year before the procedure using previously published measures (reported by Elixhauser and colleagues ${ }^{34}$ and Klabunde and colleagues, ${ }^{35}$ and prediction of severity of chronic obstructive pulmonary disease (COPD) ${ }^{36}$ ). Unknown categories were created for race, marital status, and cancer grade when patients had missing characteristics.

\section{Statistical analysis}

We performed propensity score matching separately for the primary and secondary analysis to compare patients receiving SABR with those undergoing thoracoscopic sublobar resection and lobectomy. In each analysis, we created a propensity score for receiving SABR using logistic regression based on potential confounding variables, including age, sex, race, marital status, income, residence location, tumor histology, size and stage, and comorbidities reported by Elixhauser and colleagues. ${ }^{34}$ Because SABR patients did not receive surgery and had only clinical staging, we also included pre-procedural diagnostic test (mediastinoscopy or PET scan) as a matching variable. A balanced cohort was then created by using a one-to-one nearest neighbor matching algorithm. ${ }^{37}$ The logit of the propensity score was used for matching, with a calliper width of 0.2 times its standard deviation as recommended by Austin. ${ }^{3839}$ Balance was assessed with the absolute difference (tables B and C, appendix 1).

We compared patient characteristics in the full cohort with $t$ tests for continuous variables and $\chi^{2}$ tests for categorical variables. Numbers of computed tomograms and PET scans in the first year and first two years after the procedure were compared with Wilcoxon rank sum tests in the full cohort and signed rank tests in the matched cohort.

We constructed Kaplan-Meier curves with 95\% Hall-Wellner confidence bands ${ }^{40}$ to determine overall survival and cancer specific survival. Advanced statistical techniques were used to compare difference in survival between the two treatment groups. In the main analyses with propensity score matching, we used univariable Cox proportional hazards models. For matched data, we used a univariable frailty model, which accounted for clustering of samples, to evaluate difference in survival between groups, treating paired patients as clusters. ${ }^{41}$ Proportional hazards assumption was tested to confirm the adequacy of the model. Additional statistical methods used included multivariable Cox proportional hazards models (table $\mathrm{D}$, appendix 1), instrumental variable analysis (table E, appendix 1), and competing risk analysis for cancer specific survival with Fine and Gray models ${ }^{42}$ (table F, appendix 1) in the full cohort and propensity matched cohort.

We used instrumental variable analysis to account for unobserved confounding and a two stage residual inclusion estimation method to produce unbiased estimates in non-linear models. ${ }^{43}$ This method has been previously applied to survival analyses with SEER-Medicare data. ${ }^{445}$ Pre-procedural PET scan was used as an instrument as it was related to the treatment allocation but not related to cancer outcomes. The first stage of the model produces the predicted likelihood of treatment, and raw residuals can be calculated by subtracting the predicted likelihood of receiving SABR from the actual treatment received. The second stage uses a parametric survival model with Weibull distribution, incorporating the residual as an additional covariate as well as other covariates of patient characteristics.

Sensitivity analysis was performed with Klabunde comorbidity index ${ }^{35}$ and with variables predicting severity of COPD incorporated in the propensity score matching (table G, appendix 1). Subgroup analyses were performed with the same matching strategies to compare SABR with thoracoscopic wedge and segmental resections separately (table $\mathrm{H}$, appendix 1 ). Two additional sets of analyses were conducted to compare SABR with open sublobar resection for tumor $\leq 2 \mathrm{~cm}$ and to compare SABR with open resection for tumor $\leq 5 \mathrm{~cm}$ (tables I-L, appendix 1). All analyses were carried out with SAS 9.3 (SAS Institute, Cary, NC).

\section{Patient involvement}

No patients were involved in setting the research question or the outcome measures, nor were they involved in the design and implementation of the study. There are no plans to involve patients in dissemination of the results.

\section{Results}

\section{Patients}

From October 2007 to June 2012, we identified 5821 patients undergoing SABR and thoracoscopic or open resection (SABR $n=714$ (12.3\%), video assisted thoracoscopic surgery $n=2253$ (38.7\%), open surgery $\mathrm{n}=2854(49.0 \%))$. Our primary analysis included 275 and 415 patients undergoing SABR and thoracoscopic sublobar lung resection (363 (87.5\%) wedge resection and $52(12.5 \%)$ segmental resection) for tumors sized $\leq 2 \mathrm{~cm}$. Our secondary analysis included 714 and 2253 patients undergoing SABR and thoracoscopic resection (562 (24.9\%) wedge resection, 92 (4.1\%) segmental resection, and 1599 (71.0\%) lobectomy) for tumors sized $\leq 5 \mathrm{~cm}$.

\section{Primary analysis: SABR $v$ video assisted thoracoscopic} sublobar lung resection for tumors sized $\leq 2 \mathrm{~cm}$

The mean age of the cohort was 76.4. Patients undergoing SABR were older (SABR $v$ thoracoscopic sublobar lung resection 77.6 v 75.6; $\mathrm{P}<0.001$; table 1). Patients undergoing SABR had a higher prevalence of chronic pulmonary disease than those undergoing sublobar lung resection $(88.7 \% \vee 80.2 \% ; \mathrm{P}=0.003)$. In both groups most patients were women with no significant differences noted in sex, income, or residence location of the patients. The T stage, size, histological type, and grade of cancer were significantly different between the 


\begin{tabular}{|c|c|c|c|}
\hline Variable & SABR $(n=275)$ & Thoracoscopic SLR $(n=415)$ & $P$ value \\
\hline Mean (SD) age (years) & $77.6(6.3)$ & $75.6(5.8)$ & $<0.001$ \\
\hline Men & $98(35.6)$ & $161(38.8)$ & \multirow{2}{*}{0.40} \\
\hline Women & $177(64.4)$ & $254(61.2)$ & \\
\hline \multicolumn{4}{|l|}{ Race: } \\
\hline White & $241(87.6)$ & $379(91.3)$ & \multirow{2}{*}{0.12} \\
\hline Black/other/unknown & $34(12.4)$ & $36(8.7)$ & \\
\hline \multicolumn{4}{|l|}{ Marital status: } \\
\hline Unmarried & $138(50.2)$ & $180(43.4)$ & \multirow{3}{*}{0.19} \\
\hline Married (or with partner) & $126(45.8)$ & $219(52.8)$ & \\
\hline Unknown & $11(4.0)$ & $16(3.9)$ & \\
\hline \multicolumn{4}{|l|}{ Location: } \\
\hline Metropolitan & $239(86.9)$ & $368(88.7)$ & \multirow{2}{*}{0.49} \\
\hline Non-metropolitan & $36(13.1)$ & $47(11.3)$ & \\
\hline \multicolumn{4}{|l|}{ SEER registry region: } \\
\hline Northeast & $55(20.0)$ & $121(29.2)$ & \multirow{4}{*}{0.03} \\
\hline Midwest & $51(18.5)$ & $55(13.3)$ & \\
\hline South & $67(24.4)$ & $100(24.1)$ & \\
\hline West & $102(37.1)$ & $139(33.5)$ & \\
\hline \multicolumn{4}{|c|}{ Median quarter of income distribution (2000 census): } \\
\hline 1 (lowest income) & $76(27.7)$ & $88(21.3)$ & \multirow{4}{*}{0.16} \\
\hline 2 & $66(24.1)$ & $95(22.9)$ & \\
\hline 3 & 75 (27.4) & $123(29.7)$ & \\
\hline 4 & $57(20.8)$ & $108(26.1)$ & \\
\hline \multicolumn{4}{|l|}{ Tumor T stage: } \\
\hline $\mathrm{T} 1$ & $261(94.9)$ & $348(83.9)$ & \multirow{2}{*}{$<0.001$} \\
\hline $\mathrm{T} 2$ & $14(5.1)$ & $67(16.1)$ & \\
\hline \multicolumn{4}{|l|}{ Tumor histology: } \\
\hline Adenocarcinoma & $144(52.4)$ & $197(47.5)$ & \multirow{3}{*}{$<0.001$} \\
\hline Squamous & $113(41.1)$ & $109(26.3)$ & \\
\hline Other & $18(6.5)$ & $109(26.3)$ & \\
\hline \multicolumn{4}{|l|}{ Tumor grade: } \\
\hline Grade I & $27(9.8)$ & $99(23.9)$ & \\
\hline Grade II & $47(17.1)$ & $185(44.6)$ & $<0001$ \\
\hline Grade III-IV & $58(21.1)$ & $101(24.3)$ & $<0.001$ \\
\hline Not determined & $143(52.0)$ & $30(7.2)$ & \\
\hline Tumor size $\leq 1 \mathrm{~cm}$ : & & & \\
\hline $0-10 \mathrm{~mm}$ & $24(8.7)$ & $103(24.8)$ & คคค० \\
\hline $11-20 \mathrm{~mm}$ & $251(91.3)$ & $312(75.2)$ & $<0.001$ \\
\hline Procedure type: & & & \\
\hline Wedge resection & - & $363(87.5)$ & - \\
\hline Segmental resection & - & $52(12.5)$ & - \\
\hline Year of procedure: & & & \\
\hline $2007-08$ & $37(13.5)$ & $113(27.2)$ & \\
\hline 2009-10 & $121(44.0)$ & $189(45.5)$ & $<0.001$ \\
\hline $2011-12$ & $117(42.5)$ & $113(27.2)$ & \\
\hline Pre-procedure PET scan or mediastinoscopy & $203(73.8)$ & $161(38.8)$ & $<0.001$ \\
\hline Comorbidities (Elixhauser34): & & & \\
\hline Coronary artery disease & $141(51.3)$ & $234(56.4)$ & 0.19 \\
\hline Hypertension & $236(85.8)$ & $355(85.5)$ & 0.92 \\
\hline Congestive heart failure & $78(28.4)$ & $94(22.7)$ & 0.09 \\
\hline Valve disease & $76(27.6)$ & $122(29.4)$ & 0.62 \\
\hline Diabetes & $92(33.5)$ & $164(39.5)$ & 0.11 \\
\hline Chronic pulmonary disease & $244(88.7)$ & $333(80.2)$ & 0.003 \\
\hline Obesity & $19(6.9)$ & $29(7.0)$ & 0.97 \\
\hline Anemia & $104(37.8)$ & $133(32.0)$ & 0.12 \\
\hline Peripheral vascular disease & $133(48.4)$ & $182(43.9)$ & 0.24 \\
\hline Chronic kidney disease & $47(17.1)$ & $57(13.7)$ & 0.23 \\
\hline Cerebrovascular disease & $76(27.6)$ & $109(26.3)$ & 0.69 \\
\hline Weight loss & $42(15.3)$ & $40(9.6)$ & 0.03 \\
\hline Neurologic disorder & $74(26.9)$ & $54(13.0)$ & $<0.001$ \\
\hline Hypothyroidism & $82(29.8)$ & $105(25.3)$ & 0.19 \\
\hline
\end{tabular}


Table 2 | Overall and cancer specific survival after stereotactic ablative radiotherapy (SABR) and thoracoscopic resection of lung cancer

\begin{tabular}{|c|c|c|}
\hline & \multicolumn{2}{|c|}{ Hazard ratio $(95 \% \mathrm{Cl}), \mathrm{P}$ value } \\
\hline & Full cohort & Matched cohort \\
\hline \multicolumn{3}{|c|}{ SABR $v$ thoracoscopic SLR, tumor size $\leq 2 \mathrm{~cm}$} \\
\hline Cancer specific survival & 1.47 (0.95 to 2.28$), 0.08$ & 1.32 (0.77 to 2.26$), 0.32$ \\
\hline \multicolumn{3}{|c|}{ SABR $v$ thoracoscopic resection tumor size $\leq 5 \mathrm{~cm}$} \\
\hline
\end{tabular}

groups. The matched cohort consisted of 201 patients in each treatment arm, and balance was achieved based on available variables (table $\mathrm{C}$, appendix 1).

Average follow-up of all patients was 2.9 years, and average follow-up of patients who survived was 3.6 years. In the full cohort, $144(52.4 \%)$ patients undergoing SABR died during follow-up, with 37 (13.5\%) dying from lung cancer; while 138 (33.3\%) patients undergoing thoracoscopic sublobar lung resection died during follow-up, with 44 (10.6\%) dying from lung cancer. Overall survival and cancer specific survival were longer in patients undergoing thoracoscopic
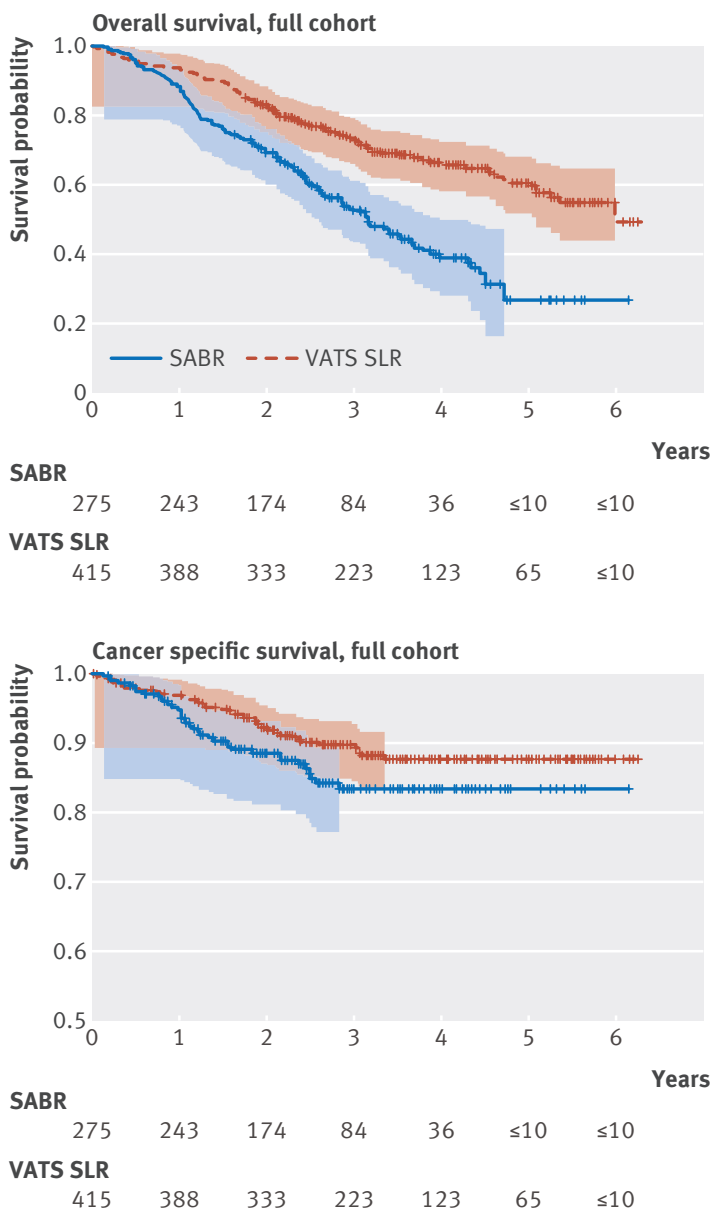

sublobar lung resection compared with patients undergoing SABR (hazard ratio 2.11 (95\% confidence interval 1.67 to $2.68, \mathrm{P}<0.001$ ) for overall survival; 1.47 (0.95 to $2.28, \mathrm{P}=0.08$ ) for cancer specific survival; table 2 ). At the end of three year follow-up estimated overall survival was $52.5 \%$ and $73.2 \%$ for patients undergoing SABR and video assisted thoracoscopic sublobar lung resection, respectively, and estimated cancer specific survival was $83.4 \%$ and $89.3 \%$, respectively (fig 1 ). In our propensity matched cohort, there was a significant difference in overall survival $(1.80,1.33$ to 2.43 ; $\mathrm{P}<0.001)$, but not in cancer specific survival (1.32, 0.77 to 2.26 ; $\mathrm{P}=0.32$ ).
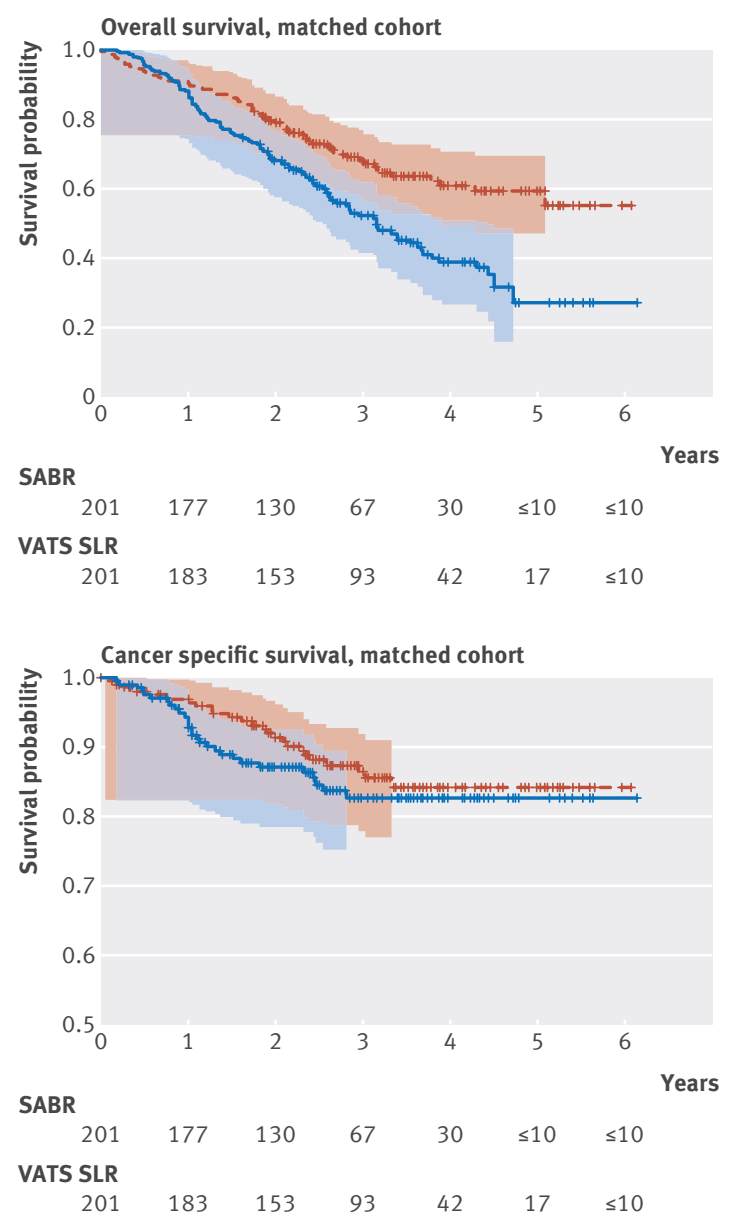

Fig 1 | Kaplan-Meier survival graphs for SABR (stereotactic ablative radiotherapy) $v$ VATS SLR (video assisted thoracoscopic sublobar lung resection) in patients with tumors sized $\leq 2 \mathrm{~cm}$ in size in full and propensity matched cohorts. Points shown with Hall-Wellner confidence bands 


\begin{tabular}{|c|c|c|c|c|c|c|}
\hline & \multicolumn{3}{|c|}{ Full cohort } & \multicolumn{3}{|c|}{ Matched cohort } \\
\hline \multicolumn{7}{|c|}{ SABR $v$ thoracoscopic SLR tumor size $\leq 2 \mathrm{~cm}$} \\
\hline PET/PET-CT in first year & $1(0-3)$ & $0(0-0)$ & $<0.001$ & $2(0-3)$ & $0(0-0)$ & $<0.001$ \\
\hline CT in first 2 years & $5(2-9)$ & $4(2-8)$ & 0.004 & $6(2-10)$ & $4(2-6)$ & $<0.001$ \\
\hline \multicolumn{7}{|c|}{ SABR $v$ thoracoscopic resection tumor size $\leq 5 \mathrm{~cm}$} \\
\hline PET/PET-CT in first year & $1(0-3)$ & $0(0-0)$ & $<0.001$ & $2(0-3)$ & $0(0-0)$ & $<0.001$ \\
\hline PET/PET-CT in first 2 years & $2(0-4)$ & $0(0-1)$ & $<0.001$ & $2(0-4)$ & $0(0-1)$ & $<0.001$ \\
\hline CT in first year & $3(2-6)$ & $2(1-4)$ & $<0.001$ & $3(2-6)$ & $2(1-4)$ & $<0.001$ \\
\hline
\end{tabular}

At three years, the estimated overall survival was 52.2\% and $68.4 \%$ for SABR and sublolar lung resection patients, and estimated cancer specific survival was $82.6 \%$ and $86.4 \%$.

Patients undergoing SABR had more follow-up imaging than those undergoing thoracoscopic sublobar lung resection (table 3). SABR patients had more chest computed tomograms as well as PET/PET-CT imaging than those undergoing sublobar lung resection in both the first year and first two years of follow-up (median $2 v 0$ PET/PET-CT $(\mathrm{P}<0.001)$ scans and $6 v 4(\mathrm{P}<0.001)$ chest computed tomograms at two years).

\section{Secondary analysis: SABR $v$ video assisted thoracoscopic resection for tumors sized $\leq 5 \mathrm{~cm}$} The mean age of the cohort was 76.0. Patients undergoing SABR were older (SABR $v$ thoracoscopic resection 78.8 v 75.2; $\mathrm{P}<0.001$; table 4). Most patients were women and white. Demographics of patients in the two treatment groups differed. Patients undergoing SABR had higher prevalence of congestive failure $(33.3 \% v 16.6 \%$; $\mathrm{P}<0.001)$ and chronic pulmonary disease $(86.7 \% v$ $69.8 \%$; $\mathrm{P}<0.001)$ before procedure. Tumor size, histological type, and grade were significantly different between the groups. The matched cohort consisted of 643 patients in each treatment arm, and balance was achieved based on available variables (table $\mathrm{D}$, appendix 1).

Average follow-up of all patients was three years, and average follow-up of survived patients was 3.7 years. Of the patients undergoing SABR, 419 (58.7\%) died during follow-up, with 119 (16.7\%) dying from lung cancer; and $680(30.1 \%)$ patients undergoing video assisted thoracoscopic resection died during follow-up, with 198 (8.8\%) dying from lung cancer. Overall survival and cancer specific survival were significantly better in patients undergoing thoracoscopic resection than in patients undergoing SABR, and significance persisted in the matched cohort (fig 2, table 2). The hazard ratio was 1.92 (95\% confidence interval 1.62 to 2.26 ; $\mathrm{P}<0.001$ ) for overall survival and 2.10 (1.52 to 2.89; $\mathrm{P}<0.001)$ for cancer specific survival. Estimated cancer specific survival at three years was $80.0 \%$ and $90.3 \%$, respectively. Similar to the finding in primary analysis, patients undergoing SABR had more follow-up imaging than those undergoing thoracoscopic resection (table 3 ).

\section{Supplemental analysis}

Supplemental analysis with multivariable regression, instrumental variable analysis, and competing risk analysis confirmed findings in our primary and secondary analysis (tables D, E, and F, appendix 1). Sensitivity analyses with Klabunde comorbidity index ${ }^{35}$ and variables predicting severity of COPD included as matching covariates were also consistent with the main analyses (table G, appendix 1). Subgroup analysis comparing SABR with thoracoscopic wedge or segment resection also showed significantly better overall survival in surgical patients and similar cancer specific survival between SABR and surgical treatment groups (table $\mathrm{H}$, appendix 1). Results from analyses comparing SABR with open surgery were also consistent with those from the main analysis (tables I-L, appendix 1).

\section{Discussion}

In this propensity matched national data analysis, we found that cancer specific survival after sublobar surgical resection was not significantly different from survival after stereotactic ablative radiotherapy (SABR) in patients with tumors sized $\leq 2 \mathrm{~cm}$. There was a tendency toward better survival after thoracoscopic sublobar lung resection. Cancer specific survival was significantly higher when thoracoscopic resection was compared with SABR in tumors sized $\leq 5 \mathrm{~cm}$. The improvement in cancer specific survival could be clinically important for patients who are younger with few other comorbid illnesses.

Other published studies that were institutional series suggested that SABR can have equivalent outcomes to surgical resection even in operable patients. ${ }^{7-14}$ The Japanese Clinical Oncology Group 0403 trial and Radiation Therapy Oncology Group trial 0618, two prospective phase II trials assessing SABR in operable stage I nonsmall cell lung cancer, have shown the overall survival to be between $76 \%$ and $85 \%$ at three years, which is similar to surgery. ${ }^{13}$ Other meta-analyses, as well as a pooled analysis of randomized phase III trials, seemed to provide some support for the equivalency of SABR compared with surgery. ${ }^{1546}$ The pooled analysis, however, was underpowered to demonstrate equivalence. ${ }^{15}$ Most of the surgical patients in these studies underwent open resection and not minimally invasive surgeries, 


\begin{tabular}{|c|c|c|c|}
\hline Variable & SABR $(n=714)$ & $\begin{array}{l}\text { Thoracoscopic } \\
\text { resection }(n=2253)\end{array}$ & $P$ value \\
\hline Mean (SD) age (years) & $78.8(6.5)$ & $75.2(5.9)$ & $<0.001$ \\
\hline Men & $277(38.8)$ & $950(42.2)$ & \multirow{2}{*}{0.11} \\
\hline Women & $437(61.2)$ & $1303(57.8)$ & \\
\hline \multicolumn{4}{|l|}{ Race: } \\
\hline White & $620(86.8)$ & $2014(89.4)$ & \multirow{2}{*}{0.06} \\
\hline Black/other/unknown & $94(13.2)$ & $239(10.6)$ & \\
\hline \multicolumn{4}{|l|}{ Marital status: } \\
\hline Unmarried & $368(51.5)$ & $891(39.5)$ & \multirow{3}{*}{$<0.001$} \\
\hline Married (or with partner) & $313(43.8)$ & $1270(56.4)$ & \\
\hline Unknown & $33(4.6)$ & $92(4.1)$ & \\
\hline \multicolumn{4}{|l|}{ Location: } \\
\hline Metropolitan & $603(84.5)$ & $2013(89.3)$ & \multirow{2}{*}{$<0.001$} \\
\hline Non-metropolitan & $111(15.5)$ & $240(10.7)$ & \\
\hline \multicolumn{4}{|l|}{ SEER registry region: } \\
\hline Northeast & $133(18.6)$ & $674(29.9)$ & \multirow{4}{*}{$<0.001$} \\
\hline Midwest & $146(20.4)$ & $185(8.2)$ & \\
\hline South & $191(26.8)$ & $594(26.4)$ & \\
\hline West & $244(34.2)$ & $800(35.5)$ & \\
\hline \multicolumn{4}{|c|}{ Median quarter of income distribution (2000 census): } \\
\hline 1 (lowest income) & $200(28.1)$ & $446(19.8)$ & \multirow{4}{*}{$<0.001$} \\
\hline 2 & $172(24.2)$ & $484(21.5)$ & \\
\hline 3 & $199(28.0)$ & $558(24.8)$ & \\
\hline 4 & $140(19.7)$ & $759(33.8)$ & \\
\hline \multicolumn{4}{|l|}{ Tumor T stage: } \\
\hline $\mathrm{T} 1$ & $499(69.9)$ & $1506(66.8)$ & \multirow{2}{*}{0.13} \\
\hline $\mathrm{T} 2$ & $215(30.1)$ & $747(33.2)$ & \\
\hline \multicolumn{4}{|l|}{ Tumor histology: } \\
\hline Adenocarcinoma & $348(48.7)$ & $1097(48.7)$ & \multirow{3}{*}{$<0.001$} \\
\hline Squamous & $310(43.4)$ & $608(27.0)$ & \\
\hline Other & $56(7.8)$ & $548(24.3)$ & \\
\hline Tumor grade: & & & \\
\hline Grade I & $76(10.6)$ & 479 (21.3) & \\
\hline Grade II & $130(18.2)$ & $1045(46.4)$ & \\
\hline Grade III-IV & $175(24.5)$ & $620(27.5)$ & $<0.001$ \\
\hline Not determined & $333(46.6)$ & $109(4.8)$ & \\
\hline Tumor size: & & & \\
\hline $0-20 \mathrm{~mm}$ & $275(38.5)$ & $1187(52.7)$ & \\
\hline $21-30 \mathrm{~mm}$ & $267(37.4)$ & $678(30.1)$ & $<0.001$ \\
\hline $31-50 \mathrm{~mm}$ & $172(24.1)$ & $388(17.2)$ & \\
\hline Procedure type: & & & \\
\hline Wedge resection & - & $562(24.9)$ & \\
\hline Segmental resection & - & $92(4.1)$ & - \\
\hline Lobectomy & - & $1599(71.0)$ & \\
\hline Year of procedure: & & & \\
\hline $2007-08$ & $97(13.6)$ & $527(23.4)$ & \\
\hline $2009-10$ & $324(45.4)$ & 1079 (47.9) & $<0.001$ \\
\hline $2011-12$ & $293(41.0)$ & $647(28.7)$ & \\
\hline Pre-procedure PET scan or mediastinoscopy & $540(75.6)$ & $1216(54.0)$ & $<0.001$ \\
\hline Comorbidities (Elixhauser ${ }^{34}$ ): & & & \\
\hline Coronary artery disease & $366(51.3)$ & $1083(48.1)$ & 0.14 \\
\hline Hypertension & $629(88.1)$ & $1904(84.5)$ & 0.02 \\
\hline Congestive heart failure & $238(33.3)$ & $373(16.6)$ & $<0.001$ \\
\hline Valve disease & $212(29.7)$ & $662(29.4)$ & 0.87 \\
\hline Diabetes & $250(35.0)$ & $759(33.7)$ & 0.51 \\
\hline Chronic pulmonary disease & $619(86.7)$ & $1572(69.8)$ & $<0.001$ \\
\hline Obesity & $48(6.7)$ & $144(6.4)$ & 0.75 \\
\hline Anemia & $280(39.2)$ & $638(28.3)$ & $<0.001$ \\
\hline Peripheral vascular disease & $330(46.2)$ & $858(38.1)$ & $<0.001$ \\
\hline Chronic kidney disease & $135(18.9)$ & $228(10.1)$ & $<0.001$ \\
\hline Cerebrovascular disease & $193(27.0)$ & $579(25.7)$ & 0.48 \\
\hline Weight loss & $92(12.9)$ & $204(9.1)$ & 0.003 \\
\hline Neurologic disorder & $199(27.9)$ & $303(13.4)$ & $<0.001$ \\
\hline Hypothyroidism & $199(27.9)$ & $599(26.6)$ & 0.50 \\
\hline
\end{tabular}



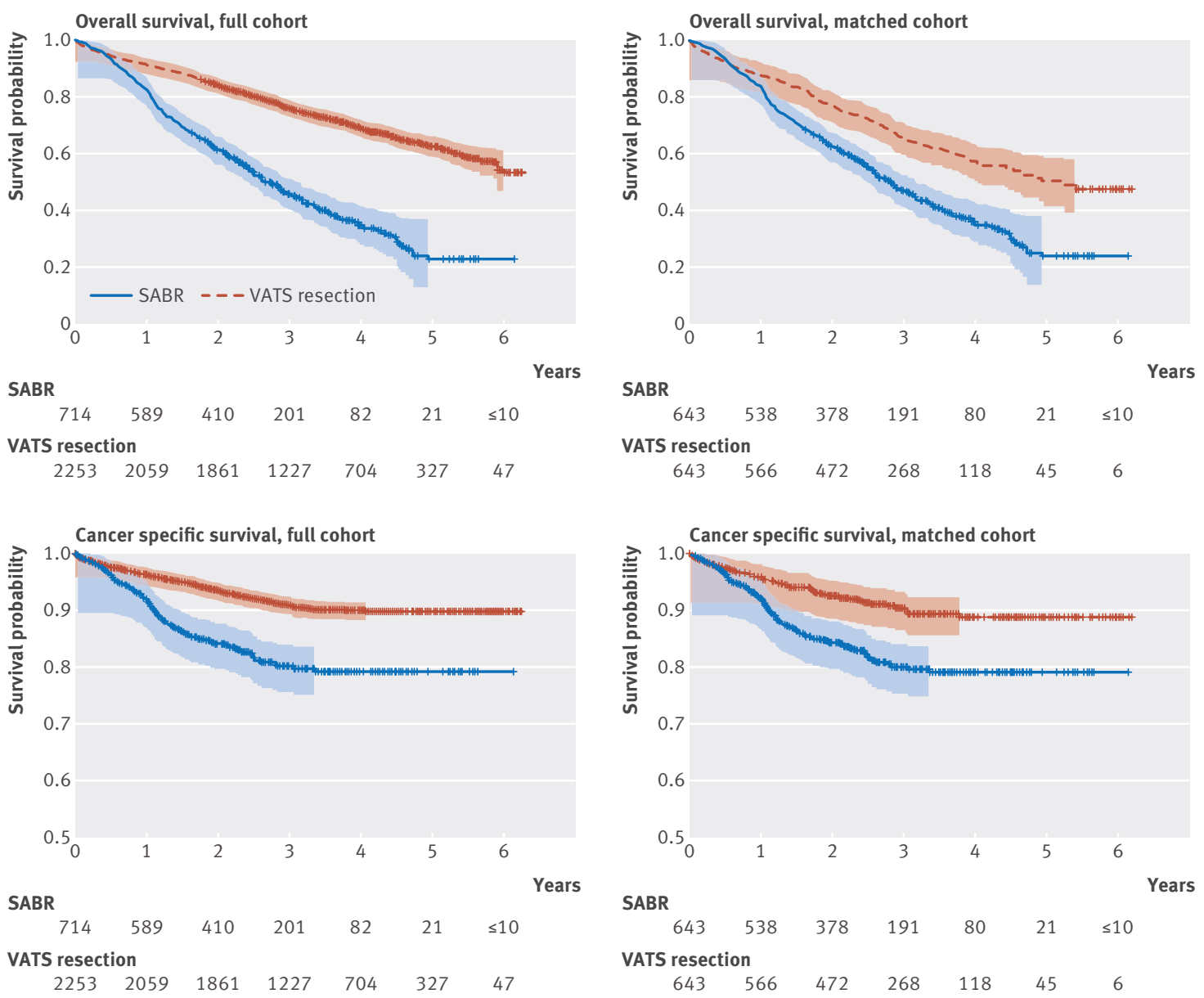

Fig 2 | Kaplan-Meier survival graphs for SABR (stereotactic ablative radiotherapy) $v$ VATS resection (video assisted thoracoscopic resection) in patients with tumors sized $\leq 5 \mathrm{~cm}$ in full and propensity matched cohorts. Points shown with Hall-Wellner confidence bands

such as video assisted thoracoscopic surgery, which are known to be associated with less morbidity and better outcomes. ${ }^{51819}$ Many of these studies also compared SABR with lobectomy rather than sublobar (wedge or segmental) resections for very early disease in patients with non-small cell lung cancer $\leq 2 \mathrm{~cm} .{ }^{547}$ Several randomized studies, including NCT00499330 (CALGB140503), are currently underway to determine the comparative effectiveness of these two approaches. Our study is the first to use a major national cohort to compare SABR with minimally invasive (thoracoscopic) sublobar resection, a popular choice for surgeons operating on patients with compromised function who are not considered able to tolerate a lobectomy.

\section{Implications of findings}

If our findings hold true in larger randomized studies, patients and providers could find that the modest benefit of surgery might not be enough to justify the associated morbidity. Surgery requires an inpatient stay, incisions, and placement of a chest tube, while SABR is delivered in outpatient settings with no incisions. Our analysis helps form the basis of future trials comparing these two methods and argues that minimally invasive techniques should be included in the trial in significant numbers to compare SABR with the least invasive surgical techniques. Otherwise, surgeons and other providers might not accept the conclusions from these trials and might negate the findings of studies of SABR versus surgery because of the lack of inclusion of minimally invasive surgery. A randomized controlled trial would be ideal to answer these and other unresolved questions regarding SABR, and several are in planning stages. It would be in the best interests of both patients and providers alike to ensure the completion of these studies with sufficient numbers of patients undergoing minimally invasive surgical techniques to facilitate their acceptance by surgeons and other providers. STABLEMATES, with its modified randomization scheme in which patients declining randomization are placed in an observational arm (sublobar resection or SABR), could serve as a model to aid recruitment as well as enrolment in these trials to ensure completion so that meaningful analyses are completed. ${ }^{28}$

Our analysis also showed that most deaths were not associated with cancer and were probably linked with age related comorbidities. The absolute difference between the treatment arms was greatest for overall survival and smaller for cancer specific measures of survival. Most patients were clearly dying from reasons 
other than their lung cancer. SABR could be an ideal choice for those who are significantly older or with numerous comorbidities such that their life expectancy is limited.

Follow-up imaging was used more frequently after SABR than after surgery, especially with respect to PET/ PET-CT. It is unclear if this leads to improved outcomes, but it certainly raises healthcare costs and requires proper economic analysis to document the cost effectiveness of SABR. A randomized controlled trial should also include economic analyses and patient preference analyses for comprehensive assessment of benefits and harms.

\section{Limitations of the study}

We recognize that there were several limitations to our analysis. First and foremost, this was not a randomized controlled trial, and there were inherent selection biases that can be controlled for but never completely eliminated. We attempted to account for apparent biases in our propensity matching. To account for differences between the two groups that were not known, such as surgeon, institutional experience, and patient's functional status, we implemented a set of instrumental variable analyses. Also, we used clinical stage for SABR patients while pathologic staging was used for patients undergoing surgical resection. SEER-Medicare does not provide clinical stage for surgical patients. We attempted to account for this using pre-procedural PET and mediastinoscopy. Bias can, however, be introduced with SABR being understaged. Given the poor state of lymph node sampling in the US for patients undergoing surgical resection (641 (13\%) patients had no lymph nodes biopsied in our analysis), surgical patients were also likely to be understaged in our study. These countering effects could in turn possibly balance each other. There was also no database variable that can substitute for a physician's estimation of risk. We attempted to compensate using variables for severity of COPD as well as a Klabunde index ${ }^{35}$ but limitations remain. As cause of death is often difficult to determine, any cause specific survival, such as cancer specific survival, is difficult to determine in general. We used cancer specific survival in this study, which is reliant on the quality of the SEER-Medicare data. Also, the overall quality of postoperative care and surveillance cannot be extrapolated from SEER-Medicare data; nor can patient preferences and quality of life, which might be considerably different between the two substantially different treatments: SABR, an outpatient procedure with no incisions versus SLR, an inpatient procedure involving incisions and a chest tube.

SABR is clearly a useful treatment for inoperable tumors. The application of SABR becomes contentious when it is used as a definitive treatment of non-small cell lung cancer staged as curable with surgery. ${ }^{31}$ It is in fact more difficult to fairly test a new technology in the face of a well established existing treatment that has come to be regarded as the ideal than if there was no previous effective treatment. Contrary to recent published reports, our population based analysis of SEER-Medicare data with its inherent limitations shows that patients undergoing thoracoscopic sublobar resection or lobectomy might have a modest improvement in cancer specific survival compared with patients undergoing SABR. Whether this benefit persists in all groups of patients or is clinically relevant is not known and requires further investigation. Given the state of ambiguity on the proper use of SABR in patients with resectable lung cancer, an adequately powered randomized controlled trial is now highly warranted to inform and ensure evidence based introduction of SABR into practice.

\section{Conclusions}

Our propensity matched analysis suggests that patients undergoing thoracoscopic surgical resection, particularly for larger tumors, might have improved cancer specific survival compared with patients undergoing SABR. Despite strategies used in study design and propensity matching analysis, there are inherent limitations to this observational analysis related to confounding, similar to most studies in healthcare that compare non-surgical technologies with surgery. As the adoption of SABR for the treatment of early stage operable lung cancer would be an important paradigm shift in lung cancer care, it warrants further thorough evaluation before widespread adoption in practice.

Contributors: SP was responsible for the study concept and design and was assisted by PL and AS. AS, IM, and All acquired the data. Al authors analyzed and interpreted the data. SP, PCL, and AS drafted the manuscript. All authors critically revised the manuscript for important intellectual content. AJI, JM, and AS were responsible for the statistical analysis. AS supervised the study and is guarantor.

Funding: AS received funding from the US FDA for establishing the MDEpiNet Science and Infrastructure Centre that enables device and surgery outcomes investigations.

Competing interests: All authors have completed the ICMJE uniform disclosure form at www.icmje.org/coi disclosure.pdf and declare: no support from any organization for the submitted work; no financial relationships with any organizations that might have an interest in the submitted work in the previous three years; no other relationships or activities that could appear to have influenced the submitted work. Ethical approval: The study was approved by the Weill Cornell Medical College institutional review board (protocol No 1308014193). Data sharing: Technical appendix and statistical code available from the corresponding author; dataset available from National Cancer Institution http://healthcaredelivery.cancer.gov/seermedicare/obtain/.

Transparency: The lead authors (study guarantors) affirm that this manuscript is an honest, accurate, and transparent account of the study being reported; that no important aspects of the study have been omitted; and that any discrepancies from the study as planned (and, if relevant, registered) have been explained.

This is an Open Access article distributed in accordance with the Creative Commons Attribution Non Commercial (CC BY-NC 3.0) license, which permits others to distribute, remix, adapt, build upon this work non-commercially, and license their derivative works on different terms, provided the original work is properly cited and the use is non-commercial. See: http://creativecommons.org/licenses/ by-nc/3.0/

Allemani C, Weir HK, Carreira H, et al. CONCORD Working Group. Global surveillance of cancer survival 1995-2009: analysis of individual data for $25,676,887$ patients from 279 population-based registries in 67 countries (CONCORD-2). Lancet 2015;385:977-1010. doi:10.1016/S0140-6736(14)62038-9.

2 Ferlay J, Soerjomataram I, Ervik M, et al. GLOBOCAN 2012 v1.0, Cancer Incidence and Mortality Worldwide: IARC CancerBase No. 11 Lyon. International Agency for Research on Cancer, 2013. http://globocan. iarc.fr. 
3 Aberle DR, Adams AM, Berg CD, et al. National Lung Screening Trial Research Team. Reduced lung-cancer mortality with low-dose computed tomographic screening. N Engl J Med 2011;365:395-409. doi:10.1056/NEJMoa1102873

4 Church TR, Black WC, Aberle DR, et al. National Lung Screening Trial Research Team. Results of initial low-dose computed tomographic screening for lung cancer. N Engl J Med 2013;368:1980-91. doi:10.1056/NEJMoa1209120.

5 Paul S, Isaacs AJ, Treasure T, Altorki NK, Sedrakyan A. Long term survival with thoracoscopic versus open lobectomy: propensity matched comparative analysis using SEER-Medicare database. BMJ 2014;349:g5575. doi:10.1136/bmj.g5575.

6 National Institute for Health and Care Excellence. Lung cancer: The diagnosis and treatment of lung cancer. NICE, 2011.

7 Chang JY, Liu H, Balter P, et al. Clinical outcome and predictors of survival and pneumonitis after stereotactic ablative radiotherapy for stage I non-small cell lung cancer. Radiat Oncol 2012;7:152. doi:10.1186/1748-717X-7-152.

8 Lagerwaard FJ, Haasbeek CJ, Smit EF, Slotman BJ, Senan S. Outcomes of risk-adapted fractionated stereotactic radiotherapy for stage I non-small-cell lung cancer. Int J Radiat Oncol Biol Phys 2008;70:68592. doi:10.1016/j.ijrobp.2007.10.053.

9 Lagerwaard FJ, Verstegen NE, Haasbeek CJ, et al. Outcomes of stereotactic ablative radiotherapy in patients with potentially operable stage I non-small cell lung cancer. Int J Radiat Oncol Biol Phys 2012;83:348-53. doi:10.1016/j.ijrobp.2011.06.2003.

10 Palma D, Visser O, Lagerwaard Fl, Belderbos J, Slotman B, Senan S. Treatment of stage I NSCLC in elderly patients: a population-based matched-pair comparison of stereotactic radiotherapy versus surgery. Radiother Oncol 2011:101:240-4 doi:10.1016/j.radonc.2011.06.029.

11 Taremi M, Hope A, Dahele M, et al. Stereotactic body radiotherapy for medically inoperable lung cancer: prospective, single-center study of 108 consecutive patients. Int J Radiat Oncol Biol Phys 2012;82:967-73. doi:10.1016/jijirobp.2010.12.039.

12 Timmerman R, Paulus R, Galvin J, et al. Stereotactic body radiation therapy for inoperable early stage lung cancer. JAMA 2010;303:1070-6. doi:10.1001/jama.2010.261.

13 Timmerman RD, Paulus R, Pass HI, et al. RTOG 0618: Stereotactic body radiation therapy (SBRT) to treat operable early-stage lung cancer patients. Proc Am Soc Clin Oncol 2013;31 (suppl):S7523.

14 Verstegen NE, Oosterhuis JW, Palma DA, et al. Stage I-II non-small-cell lung cancer treated using either stereotactic ablative radiotherapy (SABR) or lobectomy by video-assisted thoracoscopic surgery (VATS): outcomes of a propensity score-matched analysis. Ann Oncol 2013:24:1543-8. doi:10.1093/annonc/mdt026.

15 Chang JY, Senan S, Paul MA, et al. Stereotactic ablative radiotherapy versus lobectomy for operable stage I non-small-cell lung cancer: a pooled analysis of two randomised trials. Lancet Oncol 2015;16:630-7. doi:10.1016/S1470-2045(15)70168-3.

16 Crabtree TD, Denlinger CE, Meyers BF, et al. Stereotactic body radiation therapy versus surgical resection for stage I non-small cell lung cancer. J Thorac Cardiovasc Surg 2010;140:377-86. doi:10.1016/j.jtcvs.2009.12.054.

17 Shirvani SM, Jiang J, Chang JY, et al. Lobectomy, sublobar resection, and stereotactic ablative radiotherapy for early-stage non-small cell lung cancers in the elderly. JAMA Surg 2014;149:1244-53. doi:10.1001/jamasurg.2014.556.

18 Paul S, Sedrakyan A, Chiu YL, et al. Outcomes after lobectomy using thoracoscopy vs thoracotomy: a comparative effectiveness analysis utilizing the Nationwide Inpatient Sample database. Eur J Cardiothorac Surg 2013:43:813-7. doi:10.1093/ejcts/ezs428.

19 Paul S, Altorki NK, Sheng S, et al. Thoracoscopic lobectomy is associated with lower morbidity than open lobectomy: a propensitymatched analysis from the STS database. J Thorac Cardiovasc Surg 2010;139:366-78. doi:10.1016/j.jtcvs.2009.08.026.

20 Onaitis MW, Petersen RP, Balderson SS, et al. Thoracoscopic lobectomy is a safe and versatile procedure: experience with 500 consecutive patients. Ann Surg 2006;244:420-5.

21 Nicastri DG, Wisnivesky JP, Litle VR, et al. Thoracoscopic lobectomy: report on safety, discharge independence, pain, and chemotherapy tolerance. J Thorac Cardiovasc Surg 2008;135:642-7. doi:10.1016/j. jtcvs.2007.09.014

22 McKenna RJ Jr, , Houck W, Fuller CB. Video-assisted thoracic surgery lobectomy: experience with 1,100 cases. Ann Thorac Surg 2006;81:421-6. doi:10.1016/j.athoracsur.2005.07.078.

23 Lee PC, Nasar A, Port JL, et al. Long-term survival after lobectomy for non-small cell lung cancer by video-assisted thoracic surgery versus thoracotomy. Ann Thorac Surg 2013;96:951-61. doi:10.1016/j. athoracsur.2013.04.104.

24 Flores RM, Park BJ, Dycoco J, et al. Lobectomy by video-assisted thoracic surgery (VATS) versus thoracotomy for lung cancer. J Thorac Cardiovasc Surg 2009.138:11-8 doi:10.1016/i.jtcvs.2009.03.030.

25 Demmy TL, Curtis JJ. Minimally invasive lobectomy directed toward frail and high-risk patients: a case-control study. Ann Thorac Surg 1999;68:194-200. doi:10.1016/S0003-4975(99)00467-1.
26 Cattaneo SM, Park BJ, Wilton AS, et al. Use of video-assisted thoracic surgery for lobectomy in the elderly results in fewer complications. Ann Thorac Surg 2008;85:231-6. doi:10.1016/i. athoracsur.2007.07.080

27 Affairs UDoV. CSP \#2005 - Veterans Affairs Lung Cancer Surgery Or Stereotactic Radiotherapy trial (VALOR 2015). http://www.research. va.gov/programs/csp/studies.cfm.

28 Health UNIo. Stereotactic Body Radiotherapy (SBRT) Versus Sublobar Resection for High-Risk Patients Non-Small Lung Cancer 2015. https://clinicaltrials.gov/ct2/show/ NCT01622621

29 Health UNIo. Radical Resection Vs. Ablative Stereotactic Radiotherapy in Patients With Operable Stage I NSCLC (POSTILV) 2016. https://clinicaltrials.gov/ct2/show/NCT01753414.

30 Health UNIo. A Study to Determine the Feasibility and Acceptability of Conducting a Phase III Randomised Controlled Trial Comparing Stereotactic Ablative Radiotherapy With Surgery in paTients With Peripheral Stage I nOn-small Cell Lung Cancer cOnsidered Higher Risk of Complications From Surgical Resection (SABRTOOTHv1) 2016. https://clinicaltrials.gov/ct2/show/NCT02629458.

31 Rice D, Sepesi B, Heymach J, Swisher S, Vaporciyan A. SABR vs surgery for NSCLC in the media. Lancet Oncol 2015;16:e422. doi:10.1016/ S1470-2045(15)00230-2.

32 SEER-Medicare. http://seer.cancer.gov/.

33 Warren JL, Klabunde CN, Schrag D, Bach PB, Riley GF. Overview of the SEER-Medicare data: content, research applications, and generalizability to the United States elderly population. Med Care 2002;40(Suppl):IV-3-18. doi:10.1097/00005650-200208001-00002.

34 Elixhauser A, Steiner C, Harris DR, Coffey RM. Comorbidity measures for use with administrative data. Med Care 1998;36:8-27. doi:10.1097/00005650-199801000-00004.

35 Klabunde CN, Potosky AL, Legler JM, Warren JL. Development of a comorbidity index using physician claims data. J Clin Epidemiol 2000:53:1258-67. doi:10.1016/S0895-4356(00)00256-0.

36 Macaulay D, Sun SX, Sorg RA, et al. Development and validation of a claims-based prediction model for COPD severity. Respir Med 2013;107:1568-77. doi:10.1016/j.rmed.2013.05.012.

37 Rassen J, Doherty M, Huang W, Schneeweiss S. Using the Pharmacoepi Toolbox in SAS Boston, 2013. http://www.drugepi.org/ wp-content/uploads/2013/10/Using_the_Pharmacoepi_Toolbox_in_ SAS_2.4.15.pdf.

38 Austin PC. Propensity-score matching in the cardiovascular surgery literature from 2004 to 2006: a systematic review and suggestions for improvement. J Thorac Cardiovasc Surg 2007;134:1128-35. doi:10.1016/j.jtcvs.2007.07.021

39 Austin PC. Optimal caliper widths for propensity-score matching when estimating differences in means and differences in proportions in observational studies. Pharm Stat 2011:10:150-61. doi:10.1002/ pst.433.

40 Hall WJ, Wellner JA. Confidence bands for a survival curve from censored data. Biometrika 1980;67:133-43. doi:10.1093/ biomet/67.1.133.

41 Vaida F, Xu R. Proportional hazards model with random effects. Stat Med 2000;19:3309-24. doi:10.1002/1097-0258(20001230) 19:24<3309::AID-SIM825>3.0.CO;2-9.

42 Fine JP, Gray RJ. A proportional hazards model for the subdistribution of a competing risk. J Am Stat Assoc 1999;94:496-509. doi:10.1080/0 1621459.1999.10474144.

43 Terza JV, Basu A, Rathouz PJ. Two-stage residual inclusion estimation: addressing endogeneity in health econometric modeling. J Health Econ 2008;27:531-43. doi:10.1016/j.jhealeco.2007.09.009.

44 Tan HJ, Norton EC, Ye Z, Hafez KS, Gore JL, Miller DC. Long-term survival following partial vs radical nephrectomy among older patients with early-stage kidney cancer. JAMA 2012;307:1629-35. doi:10.1001/jama.2012.475.

45 Gore JL, Litwin MS, Lai J, et al. Urologic Diseases in America Project. Use of radical cystectomy for patients with invasive bladder cancer. / Natl Cancer Inst 2010;102:802-11. doi:10.1093/jnci/djq121.

46 Onishi H, Shirato H, Nagata Y, et al. Stereotactic body radiotherapy (SBRT) for operable stage I non-small-cell lung cancer: can SBRT be comparable to surgery? Int / Radiat Oncol Biol Phys 2011;81:1352-8. doi:10.1016/j.ijrobp.2009.07.1751

47 Wolf AS, Richards WG, Jaklitsch MT, et al. Lobectomy versus sublobar resection for small ( $2 \mathrm{~cm}$ or less) non-small cell lung cancers. Ann Thorac Surg 2011;92:1819-25. doi:10.1016/ j.athoracsur.2011.06.099.

(c) BMJ Publishing Group Ltd 2016

Appendix 1: Supplementary tables A-L Appendix 2: Supplementary figure (patient selection process) 